\title{
Comparison of I-gel with Baska Mask Airway for Controlled Ventilation in Obese Patients Undergoing Ambulatory Surgery: A Prospective Randomized Trial
}

\author{
Rehab Abdel Raof Abdel Aziz, Yasser Mohamed Osman \\ Department of Anesthesia and Surgical Intensive Care, Faculty of Medicine, Alexandria University, Alexandria, Egypt
}

Email address:

trcium2002@yahoo.com (R. A. R. A. Aziz), yasseralx@hotmail.com (Y.M. Osman)

To cite this article:

Rehab Abdel Raof Abdel Aziz, Yasser Mohamed Osman. Comparison of I-gel with Baska Mask Airway for Controlled Ventilation in Obese Patients Undergoing Ambulatory Surgery: A Prospective Randomized Trial. Journal of Anesthesiology. Vol. 5, No. 4, 2017 , pp. $29-35$. doi: $10.11648 /$ j.ja.20170504.12

Received: October 11, 2017; Accepted: October 26, 2017; Published: November 20, 2017

\begin{abstract}
The ideal supraglottic airway device must have high airway seal pressures during spontaneous and positive pressure ventilation also low resistance to the flow of gases. This study compared the utility of I-Gel with Baska Mask during general anesthesia in obese patients undergoing elective ambulatory surgeries. The study was conducted on sixty obese patients with body mass index (BMI) of $\geq 25$ and $\leq 40 \mathrm{~kg} \cdot \mathrm{m}^{2}$, ASA I, II, III, 18-60 years old of either sex scheduled for short duration $(<3 \mathrm{hr}$ ). I-gel was successfully inserted in 25 patients on the first attempt and in 2 patients on the second attempt (overall success rate $=90 \%$ ). Baska mask was successfully inserted in 27 patients on the first attempt and in 2 patients on the second attempt (overall success rate $=96.97 \%$ ). Successful insertion time was significantly shorter in the I-gel group. Oropharyngeal sealing pressure (OSP) was significantly higher following Baska mask insertion. Intra- and postoperative airway morbidity rates were not significantly different between both groups. So Concluded that both I-gel and Baska mask are suitable for ventilation in obese patients, but Baska mask gave a better laryngeal seal.
\end{abstract}

Keywords: I-gel, Baska Mask, Obese Patient, Ambulatory Surgery, Oropharyngeal Sealing Pressure

\section{Introduction}

General anesthetic procedure requires a safe and open airway [1]. New modalities of air way devices become available to facilitate airway manipulation. Two groups of devices, namely tracheal tube guides and supraglottic airway devices (SADs) are currently used. Supraglottic airway devices such as laryngeal mask airway and its modifications are available for use nowadays [2].

The ideal supraglottic device has to fulfill certain criteria such as high airway seal pressure with low resistance to gas flow, low incidence of pulmonary aspiration and complications. Some kinds of operations as that of day case surgeries and in laparoscopic surgeries as well as in cases of difficult airway usually necessitate the use of SADs [2, 3]. Three generations of SADs exist (table 1) [4].
Table 1. Miller's classification of SADs.

\begin{tabular}{lll}
\hline & Location of Sealing & \\
\cline { 2 - 3 } & Peri-laryngeal & Base-of-tongue \\
\hline 1st generation-inflatable cuff & cLMA, PLMA * & Combitube * \\
2nd generation-pre-shaped & I-gel ** & SLIPA * \\
3rd generation-self-energizing & Baska mask & \\
\hline
\end{tabular}

*Device has draining channel. **Device has draining channel and can be used as a conduit for blind intubation.

cLMA $=$ Classic laryngeal mask airway

PLMA $=$ Proseal laryngeal mask airway

SLIPA $=$ streamlined liner of the pharynx airway

Kanag and Meena Baska have designed Baska mask which is a supraglottic airway device without inflatable cuff and has an esophageal drainage inlet and side channels for aspiration of gastric content as well as an integrated bite-block $[9,10]$. Baska mask has all the features of other supraglottic airway devices (PLMA, LMA Supreme, I-Gel and SLIPA) [11]. In spite of the diversity of the supraglottic airways devices available, selecting a suitable one for an obese patient is 
somewhat of a challenge. The difficulty in choosing the best supraglottic device for an obese patient is due to the anatomical changes present in airway of obese patient and the high sealing pressure required due to the back pressure exerted on the respiratory system.

This study aimed at the comparison of I-Gel versus Baska Mask during general anesthesia in obese patients undergoing elective ambulatory surgeries. The primary objectives were to evaluate the devices performance, oropharyngeal sealing pressure (OSP), the ease of insertion, the success rate of insertion and the number of insertions attempts. The secondary objects were to detect the hemodynamic responses induced by airway insertion (blood pressure and heart rate), oxygen saturation, end-tidal carbon dioxide $\left(\mathrm{EtCO}_{2}\right)$ and the incidence of postoperative complications such as the presence of blood on the device, sore throat, hoarseness, and dysphagia.

\section{Methods}

\subsection{Patients and Study Design}

After approval from the Institutional Ethics Committee of the Alexandria Main University Hospitals, this study was conducted on sixty overweight and obese patients with body mass index (BMI) of $\geq 25$ and $\leq 40 \mathrm{~kg}$. $\mathrm{m}^{2}$. Patients of both sex with age ranging between 18-60 years and ASA physical status I, II, III were recruited in the study. All patients were scheduled for ambulatory surgery of short duration $(<3 \mathrm{hr})$. Written consents were obtained from all participating patients in the study. Patients with known gastrointestinal reflux, sore throat, upper respiratory airway infections were excluded. The study was registered at the Pan African Clinical Trials Registry identifier PACTR201610001791415.

History of difficult intubation was taken and the common predictive indices for difficult intubation (BMI, thyromental distance, Mallampati grade, inter-incisor distance) were measured. Patients were randomly assigned to either I-gel or Baska mask group in a 1:1 ratio using computer-generated random numbers.

\subsection{Surgical Procedure and Clinical Observations}

A multi-channel monitor was attached to all the patients for continuous electrocardiogram monitoring (ECG), noninvasive arterial blood pressure monitoring, arterial oxygen saturation $\left(\mathrm{SpO}_{2}\right)$ and capnography for end-tidal $\mathrm{CO}_{2}$ measurement. An intravenous line was secured, followed by pre-oxygenation with $100 \%$ oxygen for three minutes, then induction of anesthesia was done slowly with fentanyl 1$2 \mu \mathrm{g} / \mathrm{kg}$, propofol $2-2.5 \mathrm{mg} / \mathrm{kg}$ and neuromuscular blockade was achieved with atracurium besylate $0.5 \mathrm{mg} / \mathrm{kg}$. The patient's head was placed in the sniffing Position. Face mask ventilation was done with sevoflurane $4 \%$ in $100 \%$ oxygen for three minutes to allow full jaw relaxation. I-gel and Baska mask were lubricated and prepared.

The size of the airway device was selected based on patient's body weight in accordance with manufacturer's recommendations. For the Baska Mask, size four was used for cases between 50 to $70 \mathrm{~kg}$, size five for patients between 70 to $100 \mathrm{~kg}$, and size six for patients' over $100 \mathrm{~kg}$. In I-gel group, size 4 was used for weight between 50 and $70 \mathrm{~kg}$, and size 5 for weight more than $70 \mathrm{~kg}$.

After lubricating the I-gel, it was held firmly with its cuff outlet facing towards the chin. Then it was gently pressed down into the mouth in a direction towards the hard palate until a definitive resistance was felt. In the cases when Baska mask was used, the proximal part of the mask was compressed between the fingers and the mask was inserted in the mouth avoiding the tongue. It was pushed against the hard palate until resistance was encountered, denoting that the tip of the mask was at the upper end of the esophagus.

Appropriate placement and ventilation were determined after connecting SAD to the breathing circuit by chest wall movement, auscultation of breath sounds, a square-wave capnograph and lack of gastric insufflation. The fresh gas flow was set at $5 \mathrm{~L} / \mathrm{min}$. The patient's head was maintained in neutral position by rolls positioned bilaterally. Maintenance of anesthesia was continued with sevoflurane $(2-3 \%)$ in $35 \%$ oxygen in air. Bolus doses of $0.1 \mathrm{mg} / \mathrm{kg}$ atracurium was given for neuromuscular blockade maintenance and ventilation was initiated with tidal volume $7 \mathrm{ml} / \mathrm{kg}$ and respiratory rate (12$14 / \mathrm{min})$ was adjusted to obtain an end-tidal $\mathrm{CO}_{2}\left(\mathrm{EtCO}_{2}\right)$ between 35 and $40 \mathrm{mmHg}$. Gentle manipulation of the SAD was performed in case of inadequate ventilation. The number of attempts required for insertion was recorded. Failure of insertion was considered after second attempts and in these cases, tracheal intubation was inserted.

A well-lubricated gastric tube was inserted through the drain tube channel of either of I-gel or Baska mask. The gastric tube was left open. At the end of surgery, neuromuscular blockade was antagonized with $0.05 \mathrm{mg} / \mathrm{kg}$ neostigmine and atropine $1 \mathrm{mg}$. SAD was routinely removed after the subject has regained consciousness and adequately responds to verbal commands. The removed SAD was immediately examined for the presence or absence of blood. Perioperative complications such as laryngospasm and bronchospasm were recorded.

Apart from the demographic data and surgical procedure duration, the following data were collected: The mean arterial blood pressure (MAP), heart rate (HR), and arterial oxygen saturation $\left(\mathrm{SpO}_{2}\right)$ before and after anesthesia induction, then before SAD insertion, 1, 5, and 10 minutes after its insertion, and immediately after SAD removal. $\mathrm{EtCO}_{2}$ was measured 15 minutes after the SAD insertion, which was defined as the time between picking-up the prepared mask and successful placement. Successful placement of SAD was defined as the presence of bilateral chest expansion, a satisfactory end-tidal $\mathrm{CO}_{2}$ tracing with plateau and an estimated leak fraction of less than a third of the inspired tidal volume. The success of insertion was assessed by the number of insertion attempt, SAD failure was considered in case of failure of the second attempt despite adjustment maneuvers.

The ease of SAD insertion was qualitatively evaluated using the following 4-point scale $[5,12]$. A score 1 means 
easy insertion on first attempt with no need for adjustment, a score 2 is considered in case of slight difficult insertion on first attempt with at least one adjustment maneuver. A score 3 means obvious difficult insertion on the second attempt while score 4 is recorded in case of more than 2 attempts or no SAD insertion.

The Oropharyngeal sealing pressure (OSP) was measured once an effective airway had been achieved using a fresh gas flow rate of $5 \mathrm{~L} / \mathrm{min}$, closure of the adjustable pressure limiting valve of the anesthetic circuit and recording the pressure when gas was heard leaking around the device[5, 13]. Sore throat also was assessed as present or absent one hour after the operation and the patient was asked about the presence of dysphonia one hour and 24 hours postoperative.

\subsection{Statistical Analysis}

The previous data were statistically analysed. Unless otherwise stated, data are expressed as mean \pm standard deviation. Statistical significance was defined as $\mathrm{P}<0.05$.

\section{Results}

There were no significant differences between the two groups as regard the demographic or the surgical data (Table 2). Majority of patients were undergoing ambulatory gynecological surgery and the remainder were scheduled for breast surgery or minor surgical procedures. I-gel was successfully inserted in 25 patients $(84.3 \%)$ on the first attempt and in 2 patients on the second attempt (6.67\%) with an overall success rate of $90 \%$. Adequate ventilation was not achieved after two attempts and endotracheal tubes were inserted in 3 cases (10\%). Baska mask was successfully inserted in 27 patients $(90 \%)$ on the first attempt and in 2 patients $(6.7 \%)$ on the second attempt with an overall success rate of $96.67 \%$, (Table 3 ). Adequate ventilation was not achieved after two attempts and endotracheal tube was inserted in one patient (3.3\%).

Table 2. Demographic and surgical data.

\begin{tabular}{llll}
\hline & $\begin{array}{l}\text { I-gel group } \\
\mathbf{n}=\mathbf{3 0}\end{array}$ & $\begin{array}{l}\text { Baska mask group } \\
\mathbf{n}=\mathbf{3 0}\end{array}$ & P value \\
\hline $\begin{array}{l}\text { Age (years) } \\
\text { Range }\end{array}$ & $18-59$ & $19-56$ & 0.59 \\
Mean \pm SD & $39.6 \pm 11.09$ & $38.1 \pm 10.8$ & \\
Sex (male/ female) & $16 / 14$ & $14 / 16$ & \\
BMI & & & 0.51 \\
Range & $27-39$ & $28-39$ & \\
Mean SD & $34.1 \pm 3.35$ & $34.7 \pm 2.97$ & \\
ASAI-II-III & $5-19-6$ & $6-20-4$ & 0.23 \\
$\begin{array}{l}\text { Duration of surgery } \\
\text { (minutes) }\end{array}$ & $71 \pm 16.4$ & $53 \pm 9.98$ & \\
\hline
\end{tabular}

Table 3. Success rate of SAD insertion in both groups.

\begin{tabular}{llll}
\hline $\begin{array}{l}\text { Success rate of } \\
\text { insertion }\end{array}$ & $\begin{array}{l}\text { I-GEL group } \\
\mathbf{n = 3 0}\end{array}$ & $\begin{array}{l}\text { Baska mask group } \\
\mathbf{n = 3 0}\end{array}$ & P value \\
\hline First attempt & $25(83.3 \%)$ & $27(90 \%)$ & 0.32 \\
Second attempt & $2(6.7 \%)$ & $2(6.7 \%)$ & 0.68 \\
Failed insertion & $3(10 \%)$ & $1(3.3 \%)$ & 0.41 \\
Overall success rate & $27(90 \%)$ & $29(96,67 \%)$ & 0.058 \\
\hline
\end{tabular}

The ease of SAD insertion was comparable in patients of both groups. An easy insertion (score 1) and effective airway was achieved on the first attempt without performing adjustment maneuvers in $76.67 \%$ of Bask mask group versus $73.3 \%$ of I-gel group and no significant differences could be found (Table 4).

Table 4. Ease of SAD insertion (score) in both groups.

\begin{tabular}{|c|c|c|c|}
\hline Ease of device insertion (Scoring) & I-gel group $n=\mathbf{3 0}$ & Baska mask group $n=30$ & $P$ value \\
\hline Easy insertion (Score 1) & $22(73.3 \%)$ & $23(76.67 \%)$ & 0.47 \\
\hline Slightly difficult (Score 2) & $3(10 \%)$ & $4(13.33 \%)$ & 0.53 \\
\hline Obviously difficult (Score 3) & $2(5.7 \%)$ & $2(6.7 \%)$ & 0.58 \\
\hline Impossible (Score 4) & $3(10 \%)$ & $1(3.3 \%)$ & 0.36 \\
\hline
\end{tabular}

Insertion time for successful cases was significantly shorter in I-gel group than of Baska mask group (Table 5, Figure 1). OSP was significantly higher following Baska mask insertion than I-gel group (Table 5 and Figure 2). No significant differences were noticed between the two groups as regards $\mathrm{SpO}_{2}$ or $\mathrm{EtCO}_{2}$ (Table 5 and Figures 3, 4).

Table 5. Insertion time-Oropharyngeal sealing pressure and end-tidal carbon dioxide.

\begin{tabular}{llc}
\hline & I gel group & Baska mask group \\
\hline Insertion time (seconds)) & & $10-34$ \\
Range & $10-29$ & $19.6 \pm 8.4$ \\
Mean \pm SD & $15.6 \pm 4.6$ & 0.024 \\
Oropharyngeal leak pressure & & $23-34$ \\
Range & $21-31$ & $28.6 \pm 2.9$ \\
Mean \pm SD & $25.8 \pm 3.2$ & 0.0008 \\
End tidal carbon dioxide & & $29-41$ \\
Range & $32-41$ & $36.3 \pm 2.6$ \\
Mean \pm SD & $36.6 \pm 2.25$ & 0.63 \\
\hline
\end{tabular}




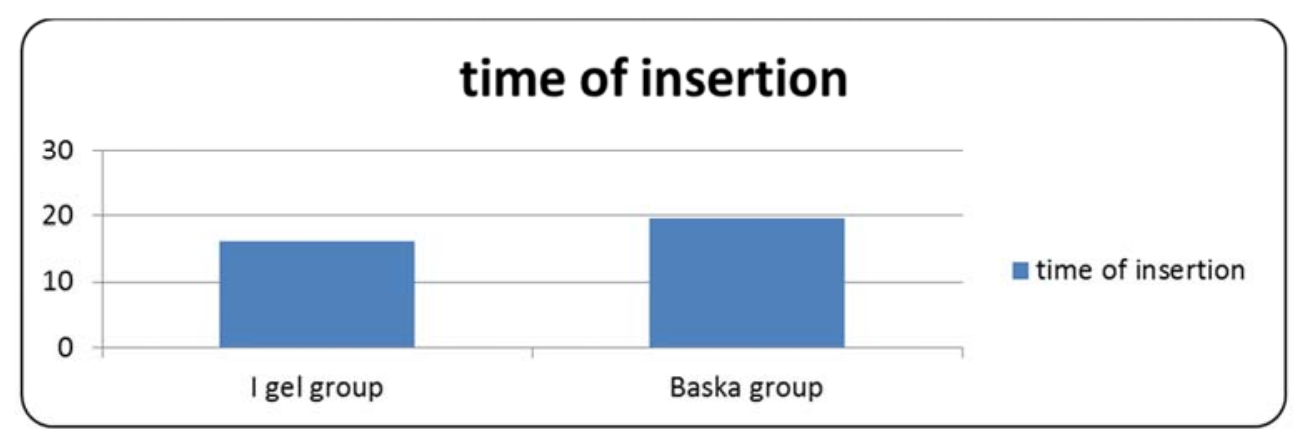

Figure 1. Changes in insertion time in the two groups.

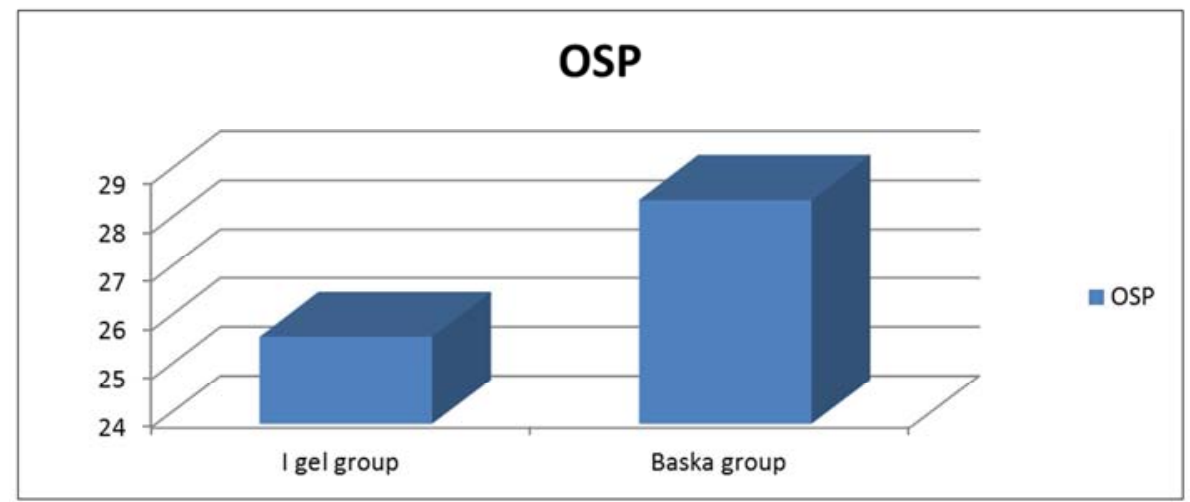

Figure 2. Changes in Oropharyngeal leak pressure $\left(\mathrm{cmH}_{2} \mathrm{O}\right)$ in the two groups.

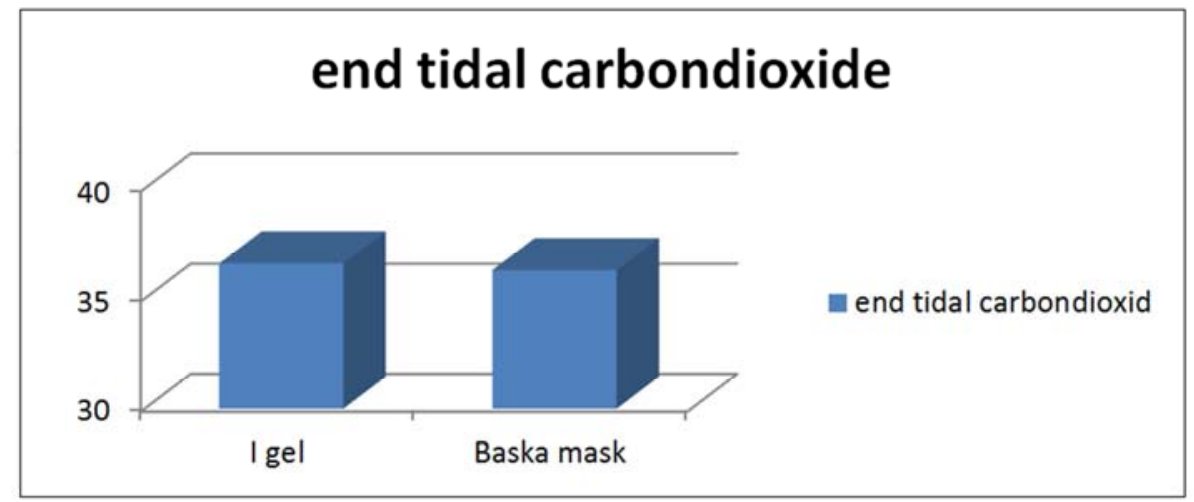

Figure 3. Changes in End tidal carbondioxide ( $\mathrm{mmHg}$ ) at 15 min in the two groups.

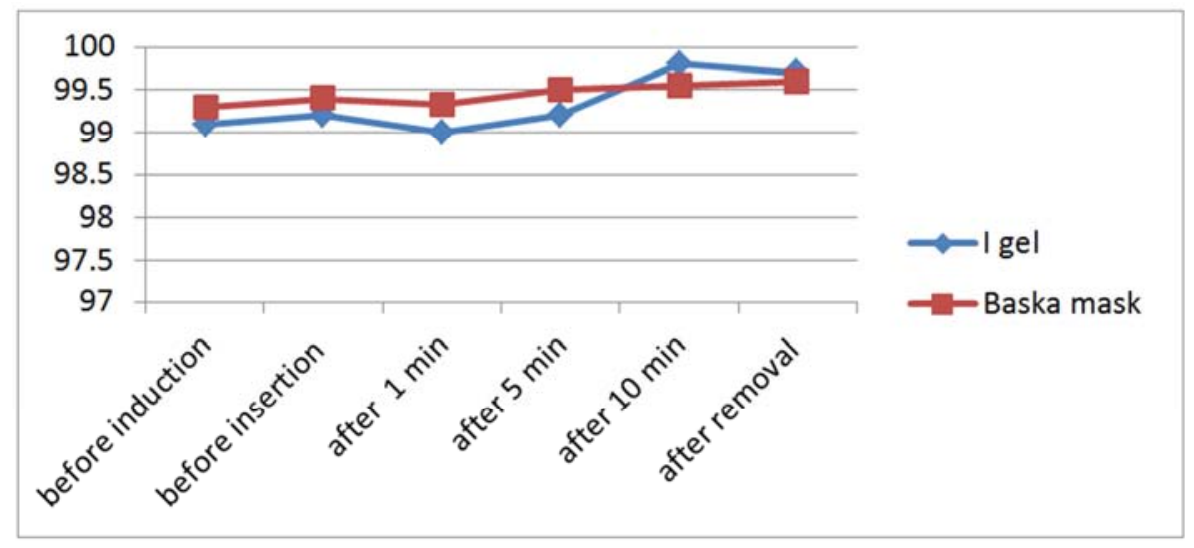

Figure 4. Changes in oxygen saturation in the two groups.

There was a significant fall in mean arterial blood pressures (MBPs) after induction compared to baseline in both groups 
(Figure 5). However, a rise in MBP occurred in patients of I -gel group after 6 minutes of device insertion compared to cases of Baska group, but no statistical difference was found. The heart rate of patients of both groups was comparable without significant difference (Figure 6).

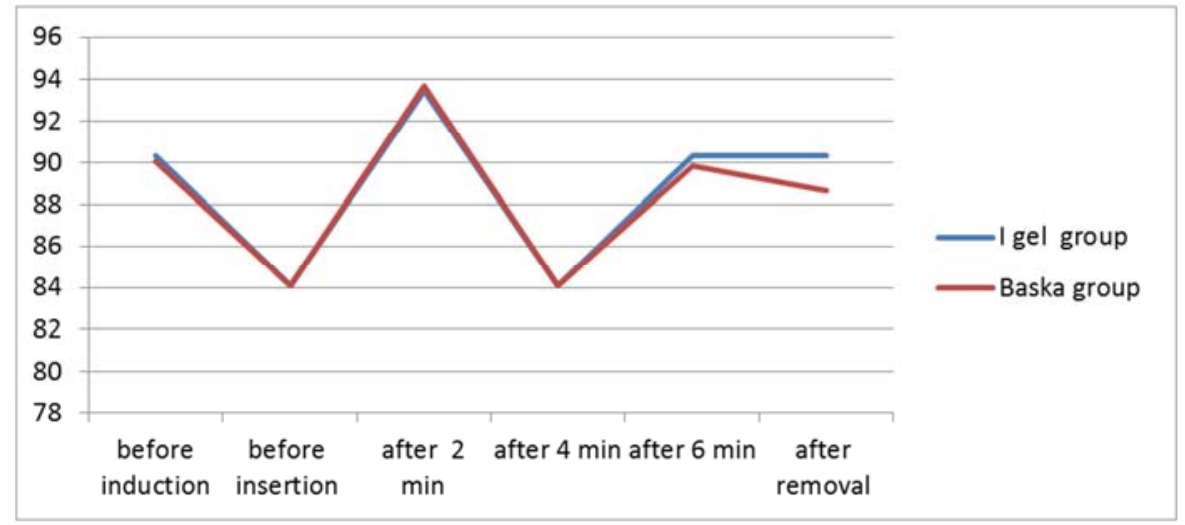

Figure 5. Changes in mean arterial blood pressure ( $\mathrm{mm} \mathrm{Hg}$ ) in the two groups.

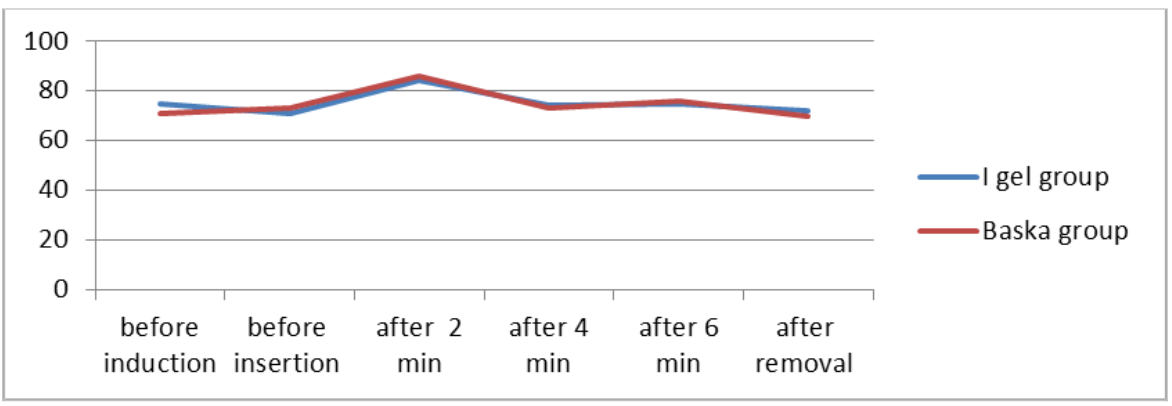

Figure 6. Changes in heart rate in the two groups.

No adverse events had occurred during surgery in any case of both groups. Blood staining was noticed in 5 cases of I-gel group ( 2 cases after the second attempt and 3 cases of failed insertion). In Baska mask group, only 3 cases in showed blood staining on device after its removal. Intra- and postoperative airway morbidity rates were insignificant between the two groups (Table 6). In I-gel group, three patients complained of sore throat after one hour of device removal and one case after 8 hours in I-gel group in comparison to a single case after one hour of device removal in Baska mask group. Only one patient complained of dysphagia in I-gel group, and no cases in Baska mask group (Table 6). Hoarseness of voice was noticed in one patient of each group after device removal.

Table 6. Incidence of postoperative complications in both groups.

\begin{tabular}{lll}
\hline & $\begin{array}{l}\text { I-gel Group } \\
\mathbf{n = 3 0}\end{array}$ & $\begin{array}{l}\text { Bask mask group } \\
\mathbf{n = 3 0}\end{array}$ \\
\hline $\begin{array}{l}\text { Blood on SAD } \\
\text { Sore throat }\end{array}$ & $5(20 \%)$ & $3(16 \%)$ \\
$1 \mathrm{hr}$ after device removal & $3(10 \%)$ & $1(3 \%)$ \\
$8 \mathrm{hr}$ after device removal & $1(3 \%)$ & $0(0 \%)$ \\
$\begin{array}{l}\text { Dysphagia } \\
1 \mathrm{hr} \text { after device removal }\end{array}$ & $1(3 \%)$ & $0(0 \%)$ \\
$8 \mathrm{hr}$ after device removal & $0(0 \%)$ & $0(0 \%)$ \\
Hoarseness, $(\%)$ & $1(3 \%)$ & $1(3 \%)$ \\
\hline
\end{tabular}

\section{Discussion}

Most of the previous studies done on supraglottic devices compared the first and second generation of SADS (I-gel with the LMA-Unique or the classic LMA) and were performed on lean not on obese patients. These studies showed that the leak pressures were higher with I-gel than with the other airway devices $[14,15,16]$. Not only the insertion times were lower but also the ease of insertion of the I-gel was better than those of the LMA-Unique in lean patients $[14,16]$.

Trivedi and Patil [17] compared the I-gel and the Proseal laryngeal mask in 60 patients ( 30 patients in each group). Igel device was successfully inserted on the first attempt in 26 patients $(86.7 \%)$ and on the second attempt in 2 patients (overall success rate was 93.3\%), failed attempts was recorded in 2 patients $(6.7 \%)$ who required endotracheal tube insertion to secure the airway.

Van Zundert and Gatt [11] in their study showed that, Baska mask was used successfully during anesthesia for surgical interventions. The device provided adequate or good outcomes in the majority of adult patients. The first attempt success rate was $88 \%$, with easy insertion rate of $92 \%$, the insertion time was short (16 seconds).

Bamgbade and colleagues [18] reported that the I-gel 
could be inserted within 5 seconds in 290 out of 300 patients, but they did not specify how insertion time was defined. Radhika, et al [19] in their study, compared LMA-S versus Igel in 42 patients were randomly divided into two groups. The first attempt success rate was higher in the LMA-S group than in the I-gel group (95\% in LMA-S group versus $85.5 \%$ in I-gel cases). They concluded that both devices are suitable for positive pressure ventilation (PPV) in anesthetized paralyzed patients. However, I-gel gave a better laryngeal seal when compared to LMA-S.

Few studies were performed on obese patients, in spite of the different anatomical challenges faced, in comparison to lean patients. Weber and his colleagues [1] compared the Igel and the LMA-Unique laryngeal mask airway in patients with mild to moderate obesity, and they found that the mean leakage pressures were $23.7 \mathrm{cmH}_{2} \mathrm{O}$ in I-gel patients versus $17.4 \mathrm{cmH}_{2} \mathrm{O}$ in LMA-Unique group. Subgroup analyses showed that the leakage pressures were higher in moderately obese group than patients with mild obesity in both I-gel and LMA-Unique groups. Insertion of I-gel was associated with significantly higher leakage pressures compared with LMA-Unique in mildly and moderately obese patients.

In order to sum all of these studies and to compare the performance of the newer second and third generation supraglottic devices (I-gel \& Baska mask) when used on obese patients we performed a study on Sixty patients with BMI $\geq 25$ and $\leq 40 \mathrm{~kg} \cdot \mathrm{mg}_{2}$. In our study, we observed significantly higher leakage pressures 28.6 $2.9 \mathrm{cmH}_{2} \mathrm{O}$ in Baska mask group compared with $25.8 \pm 3.2 \mathrm{cmH}_{2} \mathrm{O}$ in I-gel patients which is compatible with the previously discussed study of Weber [1] as regard the leakage pressures of the I-gel.

Both Baska mask and I-gel devices were easily inserted with high success rates $(90 \%$ and $83.3 \%$ on the first attempt, resp., and $96.76 \%$ and $90 \%$ overall success rate, resp.), and this finding is in agreement with the results of Trivedi [17], Van Zundert [11], Bamgbade [18] and Radhika [19] previously discussed.

The insertion of the I-gel was unsuccessful in 3 subjects because of insufficient airway sealing despite adjusted airway size; these three patients were with BMI $>35$ and $<40$ $\mathrm{kg} \cdot \mathrm{mg}^{2}$. These subjects subsequently underwent successful endotracheal intubation, in contrast to Baska mask as it was failed in one subject only also with BMI was $>35$ and $<40$. On the other hand there were no statistical difference between I-gel and Baska mask groups as regard the hemodynamic changes and the oxygen saturation in our study, this may be due to the same stress response produced by the both devices.

In this study, there was an increased no. of cases with minor blood staining of the I-gel mask after removal at the end of the surgery, but there were no differences in other complication rates, such as laryngospasm, or in the severity of throat pain, dysphagia, dysphonia or hoarseness on emergence or up to eight hours postoperatively.

The low incidence of sore throat in study can be attributed to the soft seal non inflatable mask either of I-gel or Baska mask. As these supraglottic devices without an inflatable mask have some potential advantage of easier insertion and minimal tissue compression whereas supraglottic airway devices with inflatable cuff like the LMA-Proseal can absorb anesthetic gases leading to increased mucosal pressure. This finding was in agreement with the study of Van Zundert and Gatt [11] which showed minimal blood staining and postoperative sore throat.

\section{Conclusions}

In this study, we have shown that both devices are suitable for PPV in anesthetized paralyzed patients; however Baska mask gave a better laryngeal seal when compared to I-gel and may be a better choice when used in obese patients.

\section{References}

[1] Weber U, Oguz R, Potura LA, Kimberger O, Kober A, Tschernko E. Comparison of the I -gel and the LMA-Unique laryngeal mask airway in patients with mild to moderate obesity during elective short-term surgery. Anaesthesia 2011; 66: 481-7.

[2] Almeida G, Costa AC, Machado HS. Supraglottic Airway Devices: A Review in a New Era of Airway Management. J Anesth Clin Res 2016, 7: 7 DOI: 10.4172/21556148.1000647.

[3] Ramaiah R, Das D, Bhananker SM, Joffe AM. Extraglottic airway devices: A review. International Journal of Critical Illness and Injury Science 2014; 4 (1): 77-87.

[4] Miller DM. A proposed classification and scoring system for supraglottic sealing airways: a brief review. Anesth Analg 2004; 99: 1553-1559.

[5] Radaideh K, Alhowary A, and Bani Hani D. Comparison of the Disposable Streamlined Liner of the Pharynx Airway and the Disposable I-gel in Anaesthetized, Paralyzed Adults: A Randomized Prospective Study. Anesthesiol Res Pract 2015; 2015: 971059. doi: 10.1155/2015/971059.

[6] Singh J, Yadav MK, Marahatta SB. Randomized crossover comparison of the laryngeal mask airway classic with i-gel laryngeal mask airway in the management of difficult airway in post burn neck contracture patients. Indian Journal of Anaesthesia 2012; 56(4): 348-52.

[7] Foucher-Lezla A, Lehousse T, Monrigal JP, Granry JC, and Beydon L. "Fibreoptic assessment of laryngeal positioning of the paediatric supraglottic airway device i-gel," European Journal of Anaesthesiology 2013; 30(7): 441-42.

[8] Frca TC, Frca BH. Supraglottic airway devices: recent advances. Continuing Education in Anaesthesia, Critical Care \& Pain 2011; 11 (2): 56-61.

[9] Alexiev V, Ochana A., Abdelrahman, D, Coyne J, McDonnel JGl, Toole DPO, Neligan P and Laffey JG. Comparison of the Baska mask with the single-use laryngeal mask airway in low-risk female patients undergoing ambulatory surgery. Anaesthesia 2013, 68, 1026-32.

[10] Alexiev V, Salim A, Kevin LG, Laffey JG. An observational study of the Baska_mask: a novel supraglottic airway. Anaesthesia 2012; 67: 640-5. 
[11] Zundert TV, Gatt S. The Baska Mask ${ }^{\circledR}$ - A new concept in Self-sealing membrane cuff extraglottic airway devices, using a sump and two gastric drains: A critical evaluation. Journal of Obstetric Anaesthesia and Critical Care 2012; 2 (1): 23-30.

[12] El-Radaideh K, Amarin Z, Rashdan Y, Rabadi D, Khraise W, and Omari $\mathrm{M}$. The perilaryngeal airway and the laryngeal tube in short ophthalmic procedures in adults: a prospective randomized comparative study. Journal of Anesthesia \& Clinical Research 2011; 2 (170): 1-5.

[13] Roberts M, Mani M., Wilkes A., Flavell E., and Goodwin N. A randomised crossover study comparing the disposable laryngeal mask airway Supreme with the laryngeal mask airway Proseal in unparalysed anaesthetised patients," The Internet Journal of Anesthesiology 2012; 30(2): 1-7.

[14] Francksen H, Renner J, Hanss R, Scholz J, Doerges V, Bein B. A comparison of the i-gel with the LMA Unique in nonparalysed anaesthetised adult patients. Anaesthesia 2009; 64: $1118-24$.

[15] Janakiraman C, Chethan D, Wilkes AR, Stacey MR, Goodwin
N. A randomised crossover trial comparing the i-gel supraglottic airway and classic laryngeal mask airway. Anaesthesia 2009; 64: 674-8.

[16] Uppal V, Gangaiah S, Fletcher G, Kinsella J. Randomized crossover comparison between the i-gel and the LMA-Unique in anaesthetized, paralysed adults. British Journal of Anaesthesia 2009; 103: 882-5.

[17] Trivedi Vand Patil B. A clinical comparative study of evaluation of Proseal LMA V/S I-GEL for ease of insertion and hemodynamic stability; a study of 60 cases. The Internet Journal of Anesthesiology 2009; 27(2): 1-7.

[18] Bamgbade OA, Macnab WR, Khalaf WM. Evaluation of the igel airway in 300 patients. Eur J Anaesthesiol 2008; 25: 865-6.

[19] Radhika KS, R. Sripriya, Ravishankar M, Kumar VR, Jaya V1, Parthasarathy S. Assessment of suitability of $i^{-}$-gel and laryngeal mask airway-supreme for controlled ventilation in anesthetized paralyzed patients: A prospective randomized trial. Anesth Essays Res 2016; 10 (1): 88-93. doi: 10.4103/0259-1162.167849. 